\title{
Succinate induces aberrant mitochondrial fission in cardiomyocytes through GPR91 signaling
}

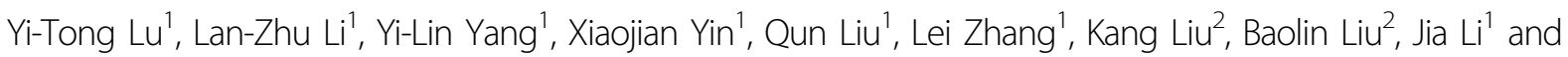 \\ Lian-Wen Qi ${ }^{1}$
}

\begin{abstract}
Altered mitochondrial metabolism acts as an initial cause for cardiovascular diseases and metabolic intermediate succinate emerges as a mediator of mitochondrial dysfunction. This work aims to investigate whether or not extracellular succinate accumulation and its targeted G protein-coupled receptor-91 (GPR91) activation induce cardiac injury through mitochondrial impairment. The results showed that extracellular succinate promoted the translocation of dynamin-related protein 1 (Drp1) to mitochondria via protein kinase C $\delta$ (PKC $\delta$ ) activation, and induced mitochondrial fission factor (MFF) phosphorylation via extracellular signal-regulated kinases-1/2 (ERK1/2) activation in a GPR91-dependent manner. As a result, enhanced localization of MFF and Drp1 in mitochondria promoted mitochondrial fission, leading to mitochondrial dysfunction and cardiomyocyte apoptosis. We further showed that inhibition of succinate release and GPR91 signaling ameliorated oxygen-glucose deprivation-induced injury in cardiomyocytes and isoproterenol-induced myocardial ischemia injury in mice. Taken together, these results showed that in response to cardiac ischemia, succinate release activated GPR91 and induced mitochondrial fission via regulation of PKC $\delta$ and ERK1/2 signaling branches. These findings suggest that inhibition of extracellular succinatemediated GPR91 activation might be a potential therapeutic strategy for protecting cardiomyocytes from ischemic injury.
\end{abstract}

\section{Introduction}

In cardiomyocytes, mitochondrial homeostasis plays a key role in maintaining heart function in response to metabolic stress ${ }^{1}$. Although inflammation, oxidative stress and endoplasmic reticulum stress are involved in cardiac injury, accumulating evidence demonstrates that mitochondrial dysfunction is an initial cause for these events $^{2,3}$. Alterations in mitochondrial morphology increases the susceptibility of the heart to ischemia/ reperfusion injury ${ }^{4,5}$, indicating the important role of

\footnotetext{
Correspondence: Jia Li (lijia0803@126.com) or L-W. Qi (Qilw@cpu.edu.cn) ${ }^{1}$ State Key Laboratory of Natural Medicines, China Pharmaceutical University, Nanjing, China

2Jiangsu Key Laboratory of TCM Evaluation and Translational Research, Department of Pharmacology of Chinese Materia Medica, China Pharmaceutical University, Nanjing, China Edited by P. Pinton
}

mitochondrial integrity in the protection of cardiac function.

Mitochondrial morphology is dynamically controlled by continuous fission and fusion. Dynamin-related protein 1 (Drp1) is a central regulator in mitochondrial fission. Drp1 is primarily located in the cytosol. Upon activation, Drp1 is recruited from the cytoplasm to the mitochondrial outer membrane, where it binds to mitochondrial fission factor (MFF) to trigger mitochondrial fission ${ }^{6}$. Drp1 activation is regulated by phosphorylation modification. Protein kinase $\mathrm{C} \delta$ (PKC $\delta$ ) and extracellular signal-regulated kinase-2 (ERK2) are shown to increase Drp1 translocation to mitochondria and promote mitochondrial fission by phosphorylation of Drp1 at Serine $616^{7,8}$. Moreover, phosphorylation of MFF may regulate the association of Drp1 with mitochondria. This concept

\section{(c) The Author(s) 2018}

(c) Open Access This article is licensed under a Creative Commons Attribution 4.0 International License, which permits use, sharing, adaptation, distribution and reproduction in any medium or format, as long as you give appropriate credit to the original author(s) and the source, provide a link to the Creative Commons license, and indicate if changes were made. The images or other third party material in this article are included in the article's Creative Commons license, unless indicated otherwise in a credit line to the material. If material is not included in the article's Creative Commons license and your intended use is not permitted by statutory regulation or exceeds the permitted use, you will need to obtain permission directly from the copyright holder. To view a copy of this license, visit http://creativecommons.org/licenses/by/4.0/. 
is supported by the enhanced binding of Drp1 to MFF when MFF is phosphorylated by AMP-activated protein kinase (AMPK) ${ }^{9}$.

Succinate is an important metabolic intermediate in the citric acid cycle and emerging evidence demonstrates that dysregulation of succinate generation is involved in cardiovascular diseases and metabolic disorders ${ }^{10}$. Although succinate is normally produced in mitochondria, the accumulated succinate can be released to extracellular space in response to pathological status, such as ischemia, diabetes and hypertension ${ }^{11,12}$. It has been demonstrated that extracellular succinate exerts a paracrine and endocrine effector through activation of its specific $\mathrm{G}$ proteincoupled receptor-91 (GPR91) ${ }^{10}$. Signaling pathways triggered by GPR91 include activation of PKC and ERK1/ $2^{13,14}$. GPR91 is highly expressed in the heart ${ }^{15}$, mediating succinate-induced cardiomyocyte death ${ }^{16}$. Moreover, upholding levels of serum succinate can cause cardiac hypertrophy through activation of GPR $91^{17}$. Given the important role of mitochondrial function in cardioprotection $^{2,3}$, this work aims to investigate whether or not extracellular succinate accumulation and its targeted GPR91 receptor activation induce cardiac injury through mitochondrial impairment.

\section{Materials and methods}

\section{Reagents and antibodies}

Sodium succinate dibasic hexahydrate, dimethyl malonate, rottlerin and tetramethylrhodamine ethyl ester perchlorate (TMRE) were purchased from Sigma-Aldrich (St Louis, MO, USA). Mito Tracker Red CMXRos (M7512) was obtained from Molecular Probes (Thermo Fisher Scientific, San Jose, CA, USA). Cell Tracker CMDil and dihydroethidium (DHE) were purchased from Beyotime Institute of Biotechnology (Shanghai, China). U0126-EtOH was purchased from Apex Bio (Houston, TX, USA). These agents were dissolved in dimethyl sulfoxide (DMSO) to obtain stock solutions and the final working concentration of DMSO was $<0.1 \%(\mathrm{v} / \mathrm{v})$. Antibodies were purchased from the following companies: anti-phospho-Drp1 (\#4494), anti-phospho-MAPK Substrates Motif [PXpTP] (\#14378), anti-Bax (\#2772) and anti-HK-II (\#2867) from Cell Signaling Technology (Beverly, MA, USA); anti-Drp1 (ab184247), antiprohibitin (ab75771), anti-PKC-delta (ab182126) and anti- $\beta$-Actin (ab8226) and anti-PKC-epsilon from Abcam (Cambridge, MA, USA); anti-MFF (sc-32577) from Santa Cruz Biotechnology, Inc. (Santa Cruz, CA, USA); antiGPR91 (BS2961), Goat Anti-Rabbit IgG (H+L), HRP (BS13278), anti-GAPDH (AP0063) and Goat Anti-Mouse IgG (H+L) (Alexa Fluor 488) (BS12478) from Bioworld Technology (St. Paul, MN, USA); Alexa Fluor 647 AffiniPure Donkey Anti-Mouse IgG $(\mathrm{H}+\mathrm{L})$ antibody from Yeasen (Shanghai, China); anti-ATP1A1 $\left(\mathrm{Na}^{+} / \mathrm{K}^{+}-\right.$
ATPase 1) (14418-1-AP) and anti-ATP5A1 (66037-1-lg) from Proteintech Group (Manchester, UK); anti-ERK1/2 (CY5487) and anti-phospho-ERK1 (T202/Y204)+ERK2 (T185/Y187) (CY5277) from Abways Technology (Shanghai, China).

\section{Animals}

Neonatal rats ( 1 or 2 days old) and ICR male mice $(18-22 \mathrm{~g})$ were purchased from the Laboratory Animal Center of Nanjing Qinglongshan. The care and treatment of animals were consistent with the Animal Ethics Committee of China Pharmaceutical University.

\section{Cell preparation and culture}

Neonatal rat ventricular myocytes (NRVMs) were prepared as previously described ${ }^{18}$. Briefly, NRVMs were isolated from 1-2-day-old Sprague-Dawley rat, digested with $0.08 \%$ collagenase and purified by differential adhesion method. Myocytes were incubated overnight in Dulbecco's modified Eagle's medium (DMEM) containing $10 \%(\mathrm{v} / \mathrm{v})$ fetal bovine serum (FBS) and $0.1 \mathrm{mmol} / \mathrm{L}$ 5-bromo-2-deoxyuridine at $37^{\circ} \mathrm{C}$ in a humidified incubator of $5 \% \quad \mathrm{CO}_{2}$ atmosphere. For oxygen-glucose deprivation (OGD) insult, the NRVMs were exposed to $1 \% \mathrm{O}_{2}$ with glucose deprivation for $4 \mathrm{~h}$. For succinate treatment, NRVMs were incubated with sodium succinate $(200 \mu \mathrm{mol} / \mathrm{L})$ for $2 \mathrm{~h}$.

H9c2 cells (American Type Culture Collection, Manassas, VA, USA) were maintained in DMEM containing $10 \%$ FBS, supplemented with antibiotics $(100 \mathrm{U} / \mathrm{mL}$ penicillin $\mathrm{G}$ and $100 \mu \mathrm{g} / \mathrm{mL}$ streptomycin sulfate), at $37^{\circ} \mathrm{C}$ in a humidified atmosphere of $5 \% \mathrm{CO}_{2}$.

\section{Isoproterenol-induced myocardial ischemic injury in mice}

Mice were treated with intraperitoneal injection of dimethyl malonate $(160 \mathrm{mg} / \mathrm{kg})$ or saline $1 \mathrm{~h}$ before isoproterenol injection $(120 \mathrm{mg} / \mathrm{kg}$, subcutaneous) for 2 consecutive days. At $24 \mathrm{~h}$ after the last injection, mice were killed for collection of hearts. For western blot analysis, left ventricle regions were lysed in ice-cold radioimmunoprecipitation assay (RIPA) buffer to extract protein. For immuofluorescent assay and apoptosis assay, the whole heart was directly fixed in $4 \%$ paraformaldehyde or $15 \%$ sucrose. The infarct size of the hearts was viewed by 2,3,5-triphenyltetrazolium chloride (TTC) staining.

\section{Measurement of intracellular $\mathrm{Ca}^{2+}$ concentration}

Intracellular $\mathrm{Ca}^{2+}$ was monitored with a $\mathrm{Ca}^{2+}$ assay kit. NRVMs were lysed with deionized water followed by 3 times of freezing and thawing cycle. After centrifuging at $10,000 \mathrm{rpm}$ for $10 \mathrm{~min}$ at $4{ }^{\circ} \mathrm{C}$, the supernatant was collected for the determination of intracellular $\mathrm{Ca}^{2+}$ contents according to the manufacturer's instructions (Jiancheng Bioengineering Institute). 


\section{Detection of reactive oxygen species (ROS)}

After stimulation with indicated agents, NRVMs were loaded with $5 \mu \mathrm{M}$ DHE working solution (Beyotime Institute of Biotechnology) at $37^{\circ} \mathrm{C}$ for $30 \mathrm{~min}$. After washing, cells were fixed in $4 \%$ paraformaldehyde for 5 min at $4{ }^{\circ} \mathrm{C}$, and ROS production was measured by a microplate reader.

\section{Measurement of succinate concentration}

After stimulation, NRVMs $\left(1 \times 10^{6}\right)$ or cardiac tissue $(10 \mathrm{mg})$ were rapidly homogenized on ice in $100 \mu \mathrm{L}$ of succinate assay buffer. Then, the homogenate was centrifuged at $10,000 \times g$ for $5 \mathrm{~min}$ to collect the supernatant. Intracellular succinate was detected using a Succinate Colorimetric Assay Kit (Sigma-Aldrich).

For measurement of extracellular succinate, NRVMs were treated with indicated agents, and then the conditional medium was collected for the assay according to the manufacturer's instructions.

\section{Membrane/cytosol fractionation}

Membrane and cytosolic fractions were prepared from NRVMs or cardiac tissues with Membrane and Cytosol Protein Extraction Kit (Beyotime Institute of Biotechnology) referring to the instructions. The samples were moderately homogenized and then centrifuged at low speed to abandon nucleus and intact cells. The supernatants were centrifuged at $14,000 \times g$ for $30 \mathrm{~min}$ at $4{ }^{\circ} \mathrm{C}$ to obtain membrane fraction. Membrane proteins were obtained with membrane protein extraction reagent. The remaining supernatant was taken as the cytosol fraction.

\section{Cytosol/mitochondria fractionation}

Cytosolic and mitochondrial fractions were prepared from NRVMs using Cell Mitochondria Isolation Kit (Beyotime Institute of Biotechnology) according to the manufacturer's protocol. Briefly, cells were collected and homogenized with a glass homogenizer in mitochondrial isolation buffer on ice. Unlysed cells and nuclei were pelleted by spinning for $10 \mathrm{~min}$ at $600 \times g$. The supernatants were centrifuged at $11,000 \times g$ for $10 \mathrm{~min}$ at $4{ }^{\circ} \mathrm{C}$ to obtain mitochondrial pellets. The remaining supernatant was taken as the cytosol fraction. Then, the mitochondrial pellets were lysed to extract mitochondrial protein using lysis buffer.

\section{Western blot analysis and immunoprecipitation (IP)}

Cells were lysed in ice-cold RIPA buffer to obtain protein by centrifugation at $12,000 \times g$ for $20 \mathrm{~min}$ at $4{ }^{\circ} \mathrm{C}$. Protein concentration was quantified using Enhanced BCA Protein Assay Kit (Beyotime Institute of Biotechnology, Shanghai, China). Equal amount of protein was separated by sodium dodecyl sulfate-polyacrylamide gel electrophoresis (SDS-PAGE), transferred onto polyvinylidene difluoride membranes and then blocked at room temperature for $2 \mathrm{~h}$. The membranes were immunoblotted with primary antibodies at $4{ }^{\circ} \mathrm{C}$ overnight. After washing, blots were incubated with horseradish peroxidase (HRP)-conjugated secondary antibody at room temperature for $2 \mathrm{~h}$. Protein bands were visualized with chemiluminescence reagent and quantified by ImageProPlus 6.0 software.

For immunoprecipitation, collected cells or cardiac tissues were incubated in the ice-cold cell lysis buffer for western and IP (Beyotime Institute of Biotechnology) for $30 \mathrm{~min}$. Cell lysates were then centrifuged at $12,000 \times g$ for $20 \mathrm{~min}$ and the soluble fraction was collected. Next, antiDrp1 antibody or anti-MFF antibody was immunoprecipitated overnight at $4{ }^{\circ} \mathrm{C}$ and then with protein $\mathrm{A}+\mathrm{G}$ agarose beads (Beyotime Institute of Biotechnology) for another $2 \mathrm{~h}$. After rinsing with the lysis buffer or phosphate-buffered saline, the beads complexes were then boiled in 1\% SDS loading buffer for western blot analysis with the indicated antibodies.

\section{Transfection of small interfering RNA}

To specifically suppress GPR91 expression, H9c2 cells of 60-80\% confluence were transfected with small interfering RNA (siRNA) duplex specific for rat GPR91 (sc-270636, Santa Cruz Biotechnology, Santa Cruz, CA, USA) or control siRNA (sc-37007) by siRNA transfection reagent (sc-29528), according to the manufacturer's instructions. For silencing of PKC $\delta$ or MFF expression, H9c2 cells were transfected with rat PKC $\delta$ siRNA or rat MFF siRNA (Genomeditech) by Lipofectamine 2000 Reagent (11668019, Thermo Fisher Scientific, San Jose, CA, USA), using control siRNA (Genomeditech) as the control. After $48 \mathrm{~h}$ post transfection, the cells were treated with sodium succinate for $2 \mathrm{~h}$ and then collected and processed for immunofluorescence microscopy and western blot.

\section{Immunofluorescence}

After treatment, NRVMs were stained with $200 \mathrm{nmol} / \mathrm{L}$ Mito Tracker Red CMXRos (Molecular Probes, Thermo Fisher Scientific, San Jose, CA, USA) to label mitochondria. NRVMs were then fixed in $4 \%$ paraformaldehyde for 20 min, and permeabilized with $0.2 \%$ Triton X-100 for 10 min at room temperature. After blocking with $3 \%$ bovine serum albumin, specimens were then labeled with specific primary antibodies overnight at $4{ }^{\circ} \mathrm{C}$ in a humidified chamber, followed by incubation with fluorescent secondary antibodies for $1 \mathrm{~h}$ at $37^{\circ} \mathrm{C}$. After washing, specimens were examined with a confocal scanning microscope (Zeiss LSM 700).

\section{Mitochondrial fission analysis}

NRVMs were stained with $200 \mathrm{nmol} / \mathrm{L}$ Mito Tracker Red CMXRos for $30 \mathrm{~min}$ at $37^{\circ} \mathrm{C}$. The structure of 
mitochondria was viewed using confocal microscopy (Zeiss LSM 700). Mitochondria with a predominantly intact network of tubular were identified as normal. Cells with disrupted and predominantly spherical mitochondria were identified as having mitochondrial fission.

To detect mitochondrial morphology in heart, cardiac tissues were fixed with $4 \%$ paraformaldehyde, dehydrated and embedded in Tissue-Tek O.C.T. Compound (Sakura Finetek, Torrance, CA, USA) and then cut into slices. The slices were stained with ATP5A1 antibody followed by secondary antibody. Mitochondrial morphology was visualized by confocal microscopy (Zeiss LSM 700).

\section{Measurement of mitochondrial membrane potential}

Treated NRVMs were loaded with the potentiometric dye TMRE $(500 \mathrm{nmol} / \mathrm{L})$ for $30 \mathrm{~min}$ at $37^{\circ} \mathrm{C}$. Fluorescence intensities were determined at the single-cell level using confocal microscopy (Zeiss LSM 700).

\section{ELISA assay of cytochrome $C$}

NRVMs were stimulated with sodium succinate $(200$ $\mu \mathrm{mol} / \mathrm{L})$ with or without mdivi-1 $(10 \mu \mathrm{mol} / \mathrm{L})$, or treated with hypoxia $\left(1 \% \mathrm{O}_{2}\right)$ in the presence or absence of dimethyl malonate $(5 \mathrm{mmol} / \mathrm{L})$. Cell cytosolic and mitochondrial fractions were collected and the concentration of cytochrome $C$ was assayed with commercial enzymelinked immunosorbent assay (ELISA) kits (R\&D, USA).

\section{Apoptosis analysis}

Cell death in the myocardium was assayed in heart paraffin section with an in situ Apoptosis Detection Kit based on the terminal deoxynucleotidyl transferasemediated dUTP nick end-labeling (TUNEL) system (Roche Applied Science, Upper Bavaria, Germany). The staining was detected by NanoZoomer 2.0-RS (Hamamatsu, Japan). In addition, cell apoptosis in NRVMs was detected with TUNEL assays kit (Vazyme Biotech Co., Nanjing, China) by a confocal scanning microscope (Zeiss LSM 700).

\section{Statistical analysis}

Data are presented as mean values $\pm \mathrm{SD}$. The significance of differences was analyzed by one-way analysis of variance followed by the Tukey's test. A value of $p<$ 0.05 is considered statistically significant.

\section{Results}

\section{Succinate stimulation activated GPR91 receptor}

It is demonstrated that an increase in intracellular $\mathrm{Ca}^{2+}$ concentration is the feature of GPR91 activation ${ }^{19}$. We observed that sodium succinate effectively increased intracellular $\mathrm{Ca}^{2+}$ contents at concentrations ranging from $100 \mu \mathrm{M}$ to $1 \mathrm{mM}$ with half-maximal effective concentration $\left(\mathrm{EC}_{50}\right)$ of $153.9 \mu \mathrm{M}$ (95\% confidence interval,
92.37-256.5 $\mu \mathrm{M}$ ) (Fig. 1a). Thus, sodium succinate was used at a working concentration of $200 \mu \mathrm{M}$ as extracellular succinate to stimulate cardiomyocytes in subsequent experiments, consistent with the previous studies $^{10,20}$. Immunofluorescent staining revealed that GPR91 was localized to the plasma membrane in cardiomyocytes under basal condition. Sodium succinate was capable of inducing GPR91 internalization (Fig. 1b), further confirming the activation of GPR91. Western blot results showed that succinate increased GPR91 expression in the cytosolic fraction, in parallel with decreased expression in membrane (Fig. 1c). PKC activation is a branch of cellular signaling in response to GPR91 activation $^{13}$. Confocal microscopy results showed that succinate induced $\mathrm{PKC} \delta$, but not $\mathrm{PKC}$, translocation to mitochondria (Fig. 1d, e). Correspondingly, succinate increased PKC $\delta$ protein expression in mitochondria (Fig. 1f). PKC $\delta$ inhibitor rottlerin prevented PKC $\delta$ translocation to mitochondria. These results indicated that extracellular succinate activated membrane GPR91 with PKC $\delta$ translocation to mitochondria.

\section{Succinate promoted Drp1 recruitment to mitochondria via GPR91-dependent PKC $\delta$ activation}

Mitochondrial dynamics is required to preserve mitochondrial function. Phosphorylation of Drp1 at Ser 585 in rat (equivalent to Ser 616 of human Drp1 isoform 1) promotes Drp1 translocation to mitochondria and induces mitochondrial fission ${ }^{21,22}$. Sodium succinate increased Drp1 phosphorylation (Ser 585) (Fig. 2a) and promoted Drp1 translocation to mitochondria (Fig. 2c, d), whereas these alterations were reversed by PKC $\delta$ inhibitor rottlerin and PKC $\delta$ knockdown with siRNA treatment (Fig. 2a-e). To determine whether or not Drp1 and PKC $\delta$ interacted directly, we isolated Drp1 protein from the cell lysate by immunoprecipitation. Increased PKC $\delta$ protein expression was observed in Drp1 protein (Fig. 2f). These results indicated that PKC $\delta$ could bind to Drp1 in the cytosol, leading to Drp1 activation. As expected, succinate promoted Drp1 recruitment and caused mitochondrial fragmentation, a morphologic change referred as mitochondrial fission (Fig. 2g). To prove the role of GPR91, we treated H9c2 cells with GPR91 siRNA, and observed that GPR91 knockdown diminished succinate-induced PKC $\delta$ translocation to mitochondria and Drp1 phosphorylation (Ser 585) (Fig. 2h, i).

\section{Succinate activated MFF through ERK1/2 in a GPR91- dependent manner}

Apart from PKC $\delta$ activation, ERK1/2 is another signaling branch from membrane GPR91 activation ${ }^{13,14}$. In cardiomyocytes, sodium succinate insult increased ERK1/ 2 phosphorylation and promoted its translocation to mitochondria (Fig. 3a, b). MFF is a receptor for Drp1 to 


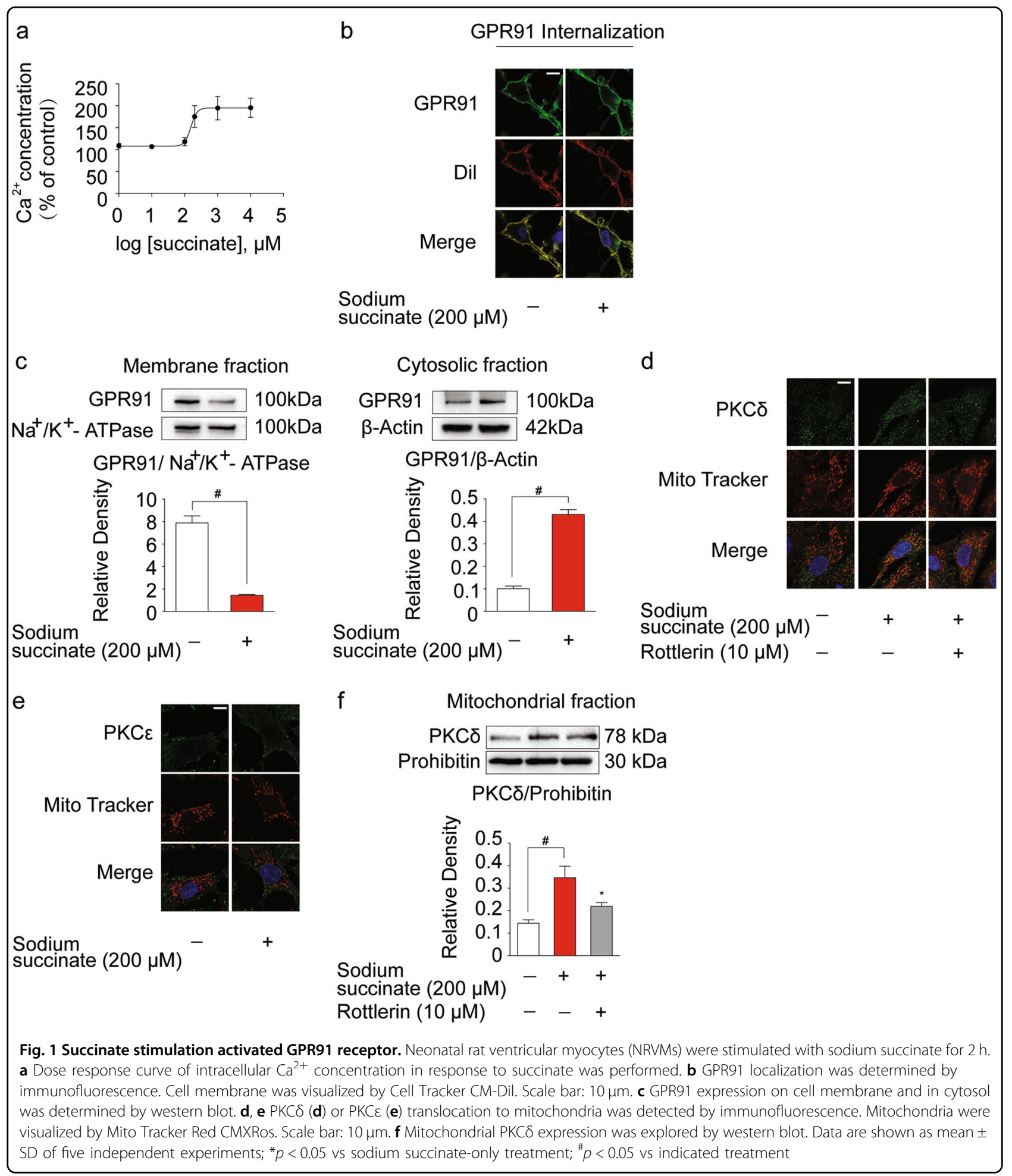

facilitate mitochondrial fission ${ }^{6}$. To identify the putative sites of MFF phosphorylated by ERK1/2, complete amino acid sequences of MFF were scanned by the Scansite databases (phosphorylation sites identification software: Group-based Prediction System, ver 3.0) for ERK1/2 consensus phosphorylation motifs. Search result revealed that rat MFF contains ERK1/2 consensus phosphorylation sites at T112 (Fig. 3c), conforming to the optimal ERK1/2 motif PXSP/PXTP ${ }^{23}$. Immunoprecipitation showed that phosphorylated ERK1/2 consensus sequence was 


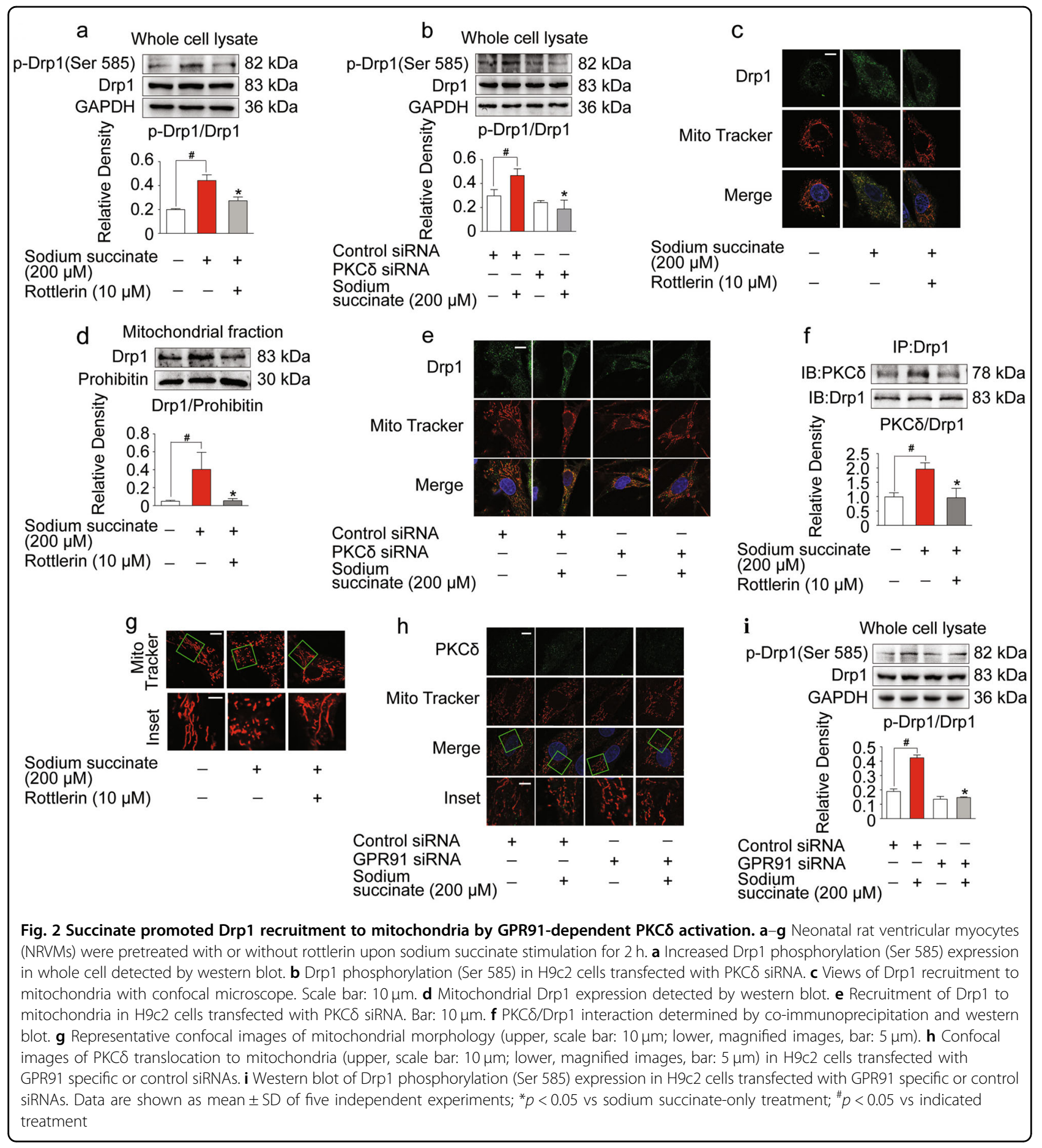

presented in MFF. Succinate treatment increased MFF phosphorylation, and this alteration was decreased by ERK1/2 inhibitor U0126-EtOH (Fig. 3d). To confirm the role of GPR91, we treated H9c2 cells with GPR91 siRNA, and observed that GPR91 knockdown diminished succinate-induced ERK1/2 phosphorylation and MFF phosphorylation (Fig. 3e, f), indicative of the involvement of GPR91 in ERK1/2 and MFF activation.

\section{Succinate induced mitochondrial fission by GPR91-} dependent Drp1 and MFF interaction

MFF is the primary receptor for Drp1 to facilitate mitochondrial fission. Co-immunoprecipitation showed that succinate increased MFF and Drp1 localization, and this effect was reduced by ERK1/2 inhibitor U0126-EtOH and PKC $\delta$ inhibitor rottlerin, respectively (Fig. 4a). Consistently, immunofluorescent staining further revealed 


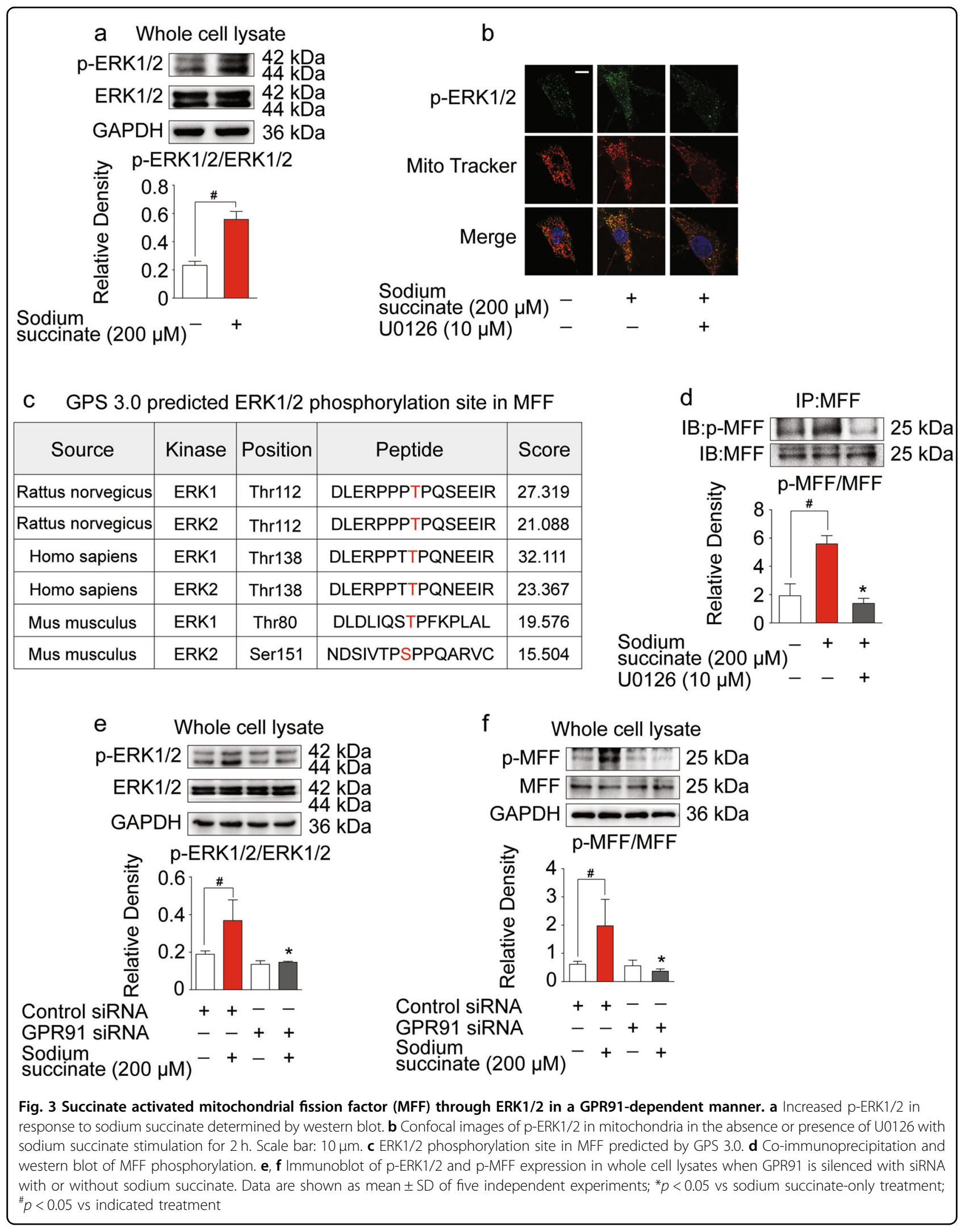




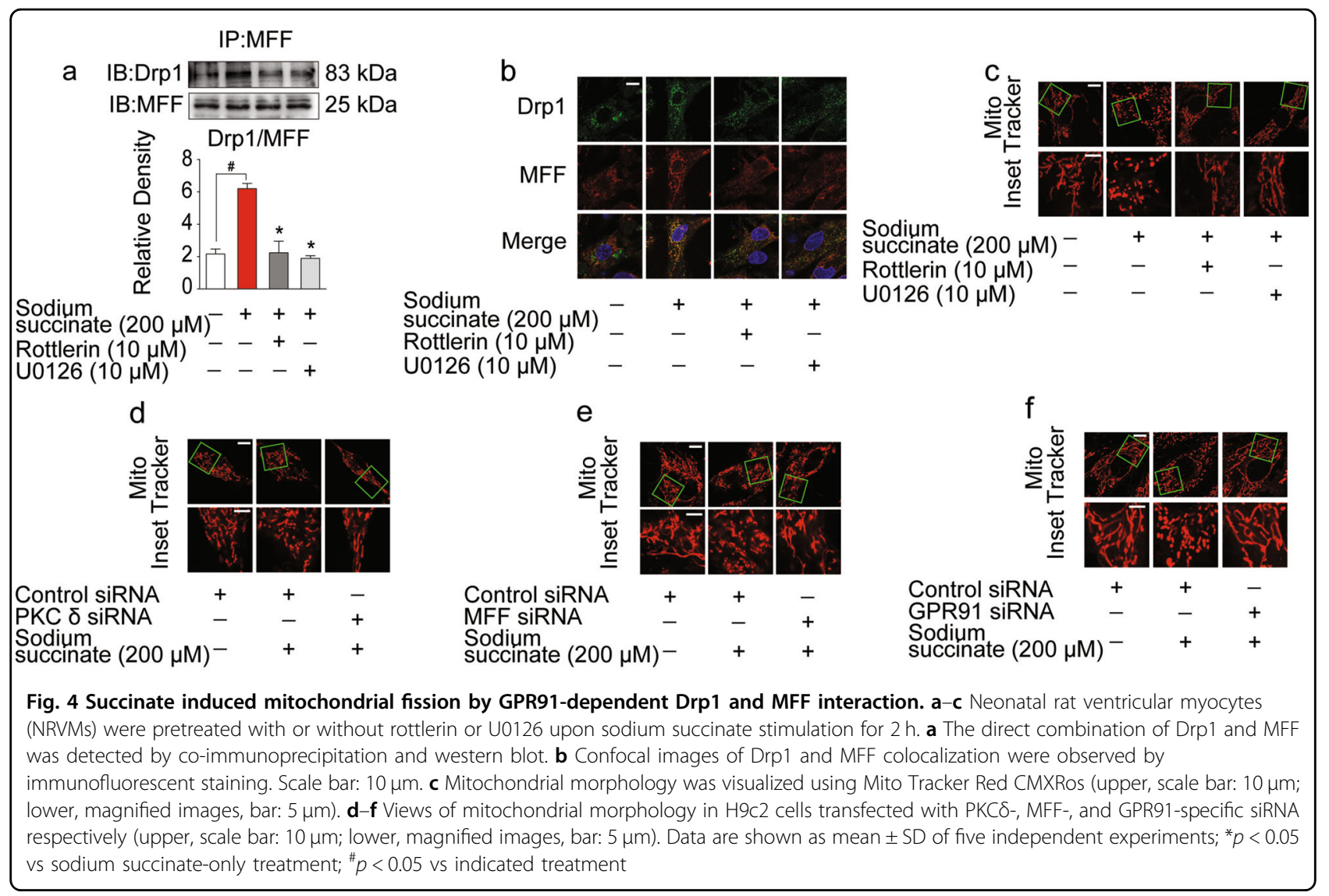

that MFF and Drp1 localization increased in response to succinate insult (Fig. 4b). As a result, succinate stimulation induced mitochondrial fission in cardiomyocytes, and this alteration was alleviated by inhibition of $\mathrm{PKC} \delta$ and ERK1/2 (Fig. 4c). Knocking down PKC $\delta$ or MFF prevented mitochondrial fission in response to succinate stimulation (Fig. 4d, e), suggesting the functional importance of PKC $\delta$ and MFF. Silencing of GPR91 prevented mitochondrial fission upon succinate treatment, confirming the indispensable role of GPR91 in succinateinduced mitochondrial fission (Fig. 4f).

\section{Succinate-stimulated mitochondrial fragmentation led to mitochondrial dysfunction and cell death}

Next, we investigated the functional consequence of succinate-induced mitochondrial integrity impairment. We observed that succinate stimulation induced the collapse of mitochondrial membrane potential, but this action was attenuated by fission inhibitor mdivi-1 (Fig. 5a). Inhibition of mitochondrial fission by mdivi-1 preserved mitochondrial hexokinase II (HK-II) (Fig. 5b). Meanwhile, against succinate insult, mdivi-1 treatment decreased Bax expression in mitochondrial fraction (Fig. 5c). As a result, mitochondrial fission inhibitor mdivi-1 limited cytochrome $C$ release from mitochondria, and subsequently reduced cell apoptosis in cardiomyocytes upon succinate insult (Fig. 5d, e). These results demonstrated that succinate impaired mitochondrial integrity and then led to mitochondrial dysfunction and cell death.

\section{Inhibition of hypoxic succinate release protected cardiomyocytes from OGD injury}

Hypoxic succinate accumulation is a result from the reversal of succinate dehydrogenase $(\mathrm{SDH})$ activation $^{24}$. $\mathrm{SDH}$ inhibitor malonate reduced succinate generation in mitochondria and thereby prevented succinate release during OGD (Fig. 6a). As expected, SDH inhibitor malonate reduced OGD-induced colocalization of MFF and Drp1 (Fig. 6b) and therefore prevented mitochondrial fission (Fig. 6c). Meanwhile, we observed that both malonate and knockdown of GPR91 reduced ROS production under OGD condition, suggesting the possibility that hypoxic succinate release induced oxidative stress with the involvement of GRP91 (Fig. 6d). Malonate pretreatment prevented the loss of mitochondrial membrane potential and reduced mitochondrial cytochrome $C$ release, resultantly reducing 


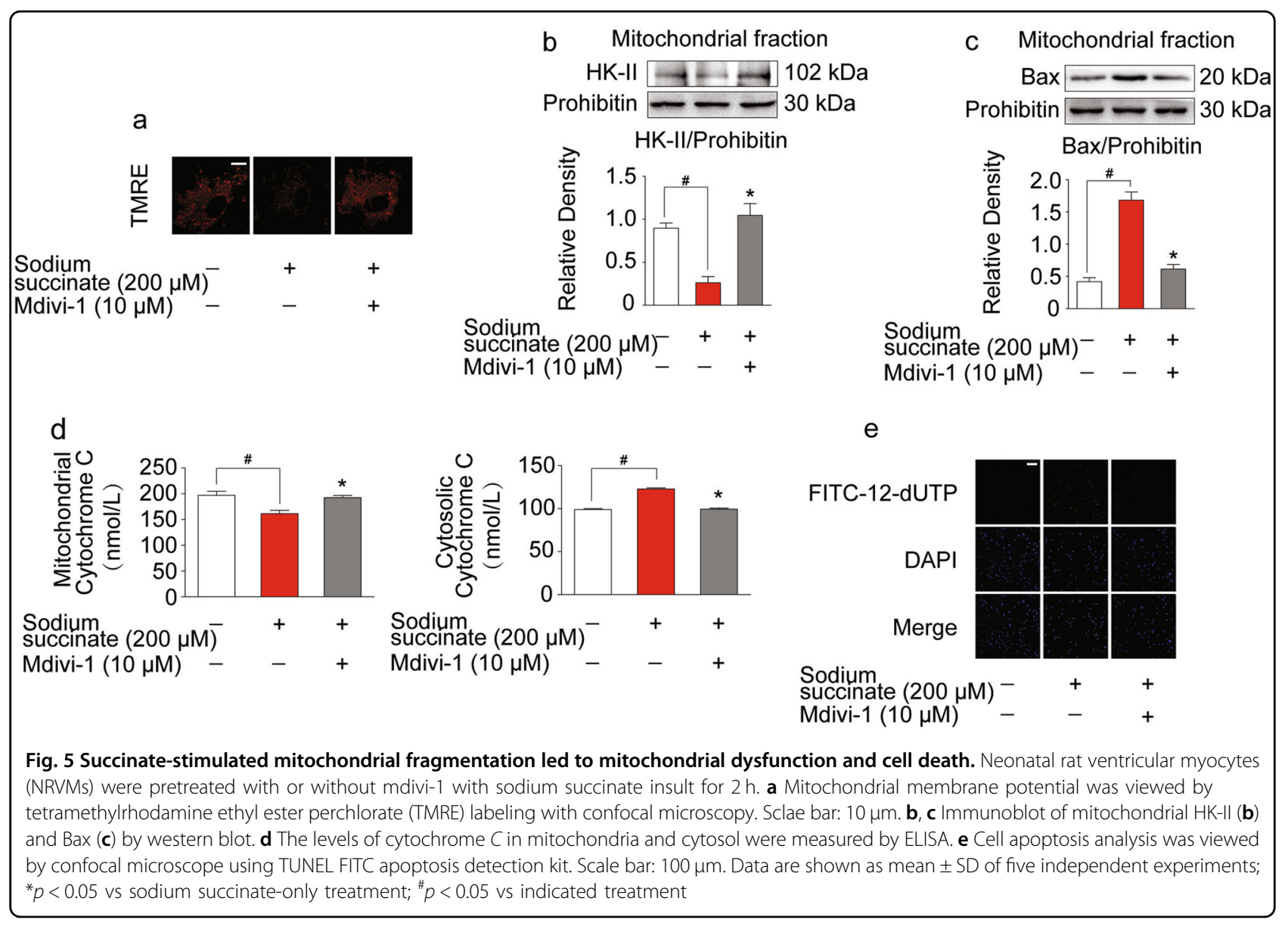

OGD-induced cell apoptosis (Fig. 6e-g). Although intracellular succinate accumulation could impair mitochondrial function through different mechanisms ${ }^{20,24}$, these results suggested that reduction of cellular succinate accumulation by SDH inhibition contributed to protecting mitochondrial function by limiting succinate release.

Inhibition of succinate accumulation protected heart from isoproterenol- induced myocardial ischemia injury in mice

To test the effects of succinate in vivo, we prepared isoproterenol-induced cardiac ischemia model in mice. We found that succinate accumulated in the ischemic heart, whereas the accumulation was reduced by malonate treatment (Fig. 7a). Malonate administration decreased GPR91 expression in the cytosolic fraction, while the expression in membrane was preserved (Fig. 7b). Because GPR91 activation is special for extracellular succinate ${ }^{10}$, these results suggested that accumulated succinate in mitochondria was released to activate membrane GPR91. Subsequently, malonate treatment attenuated colocalization of MFF and Drp1 in mitochondria and then prevented mitochondrial fission (Fig. 7c, d) in the heart. Moreover, malonate limited infarct size (Fig. 7e) and reduced apoptosis (Fig. 7f) in the heart against isoproterenol-induced ischemic injury.

\section{Discussion}

Altered mitochondrial dynamics and function are considered the initial causes for cardiac injury ${ }^{3,25}$. Intracellular succinate mediates oxidative stress due to reverse electron transport during reperfusion ${ }^{24}$; however, it remains unknown whether extracellular succinate is also implicated in ischemic injury. Herein, we showed that succinate released to extracellular space acted as a signaling molecule to mediate mitochondrial fission via GPR91 signaling pathways in a paracrine and autocrine manner. These findings provided a molecular mechanism to link metabolic dysregulation with impaired mitochondrial integrity.

Apart from being an intermediate in the citric acid cycle, succinate is regarded as a signaling molecule involved in the regulations of cellular responses, including inflammation, hypoxia, oxidative stress and tumorigenesis $^{26,27}$. Succinate is produced within mitochondria, and can translocate into the cytosol through specific 


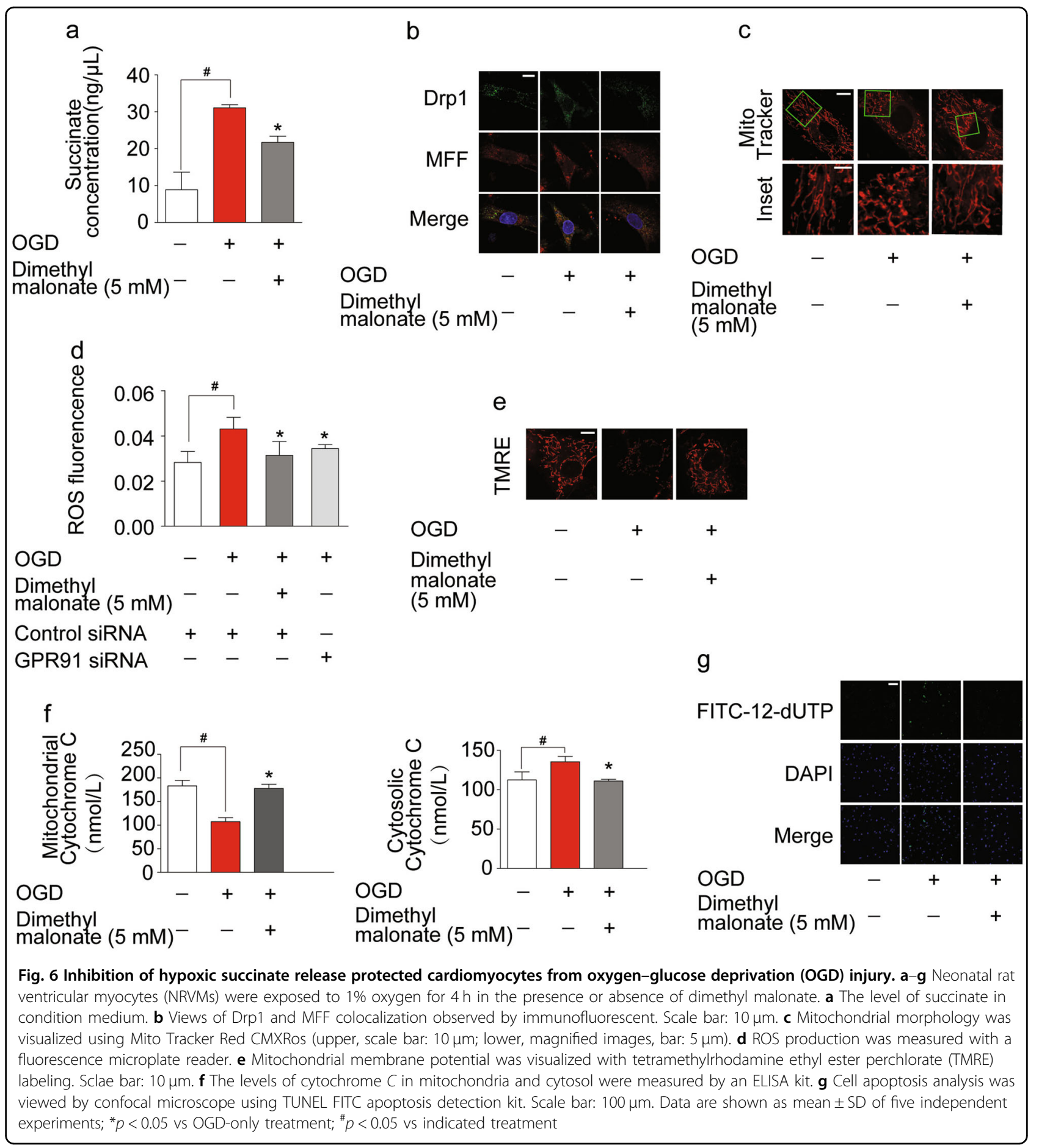

transporter SLC25A10 ${ }^{28}$, and release to the extracellular space due to local energy metabolism disturbances ${ }^{12,29,30}$. In line with these reports, we found that succinate contents in the culture medium were elevated in response to OGD and isoproterenol challenge in mice induced succinate accumulation in the ischemic heart, suggesting the possibility that the released succinate might influence cellular response in an autocrine/paracrine manner.
GPR91 is a plasma membrane receptor presenting in many tissues, including the heart ${ }^{17}$, and acts as a specific sensor for extracellular succinate ${ }^{12}$. We observed that sodium succinate stimulation activated GPR91 in cardiomyocytes, evidenced by the internalization of the receptor. GPR91 is a Gq-coupled receptor which can mediate the $\mathrm{PKC} /$ mitogen-activated protein kinase (MAPK) signaling cascades $^{12,31}$. Mitochondrial 


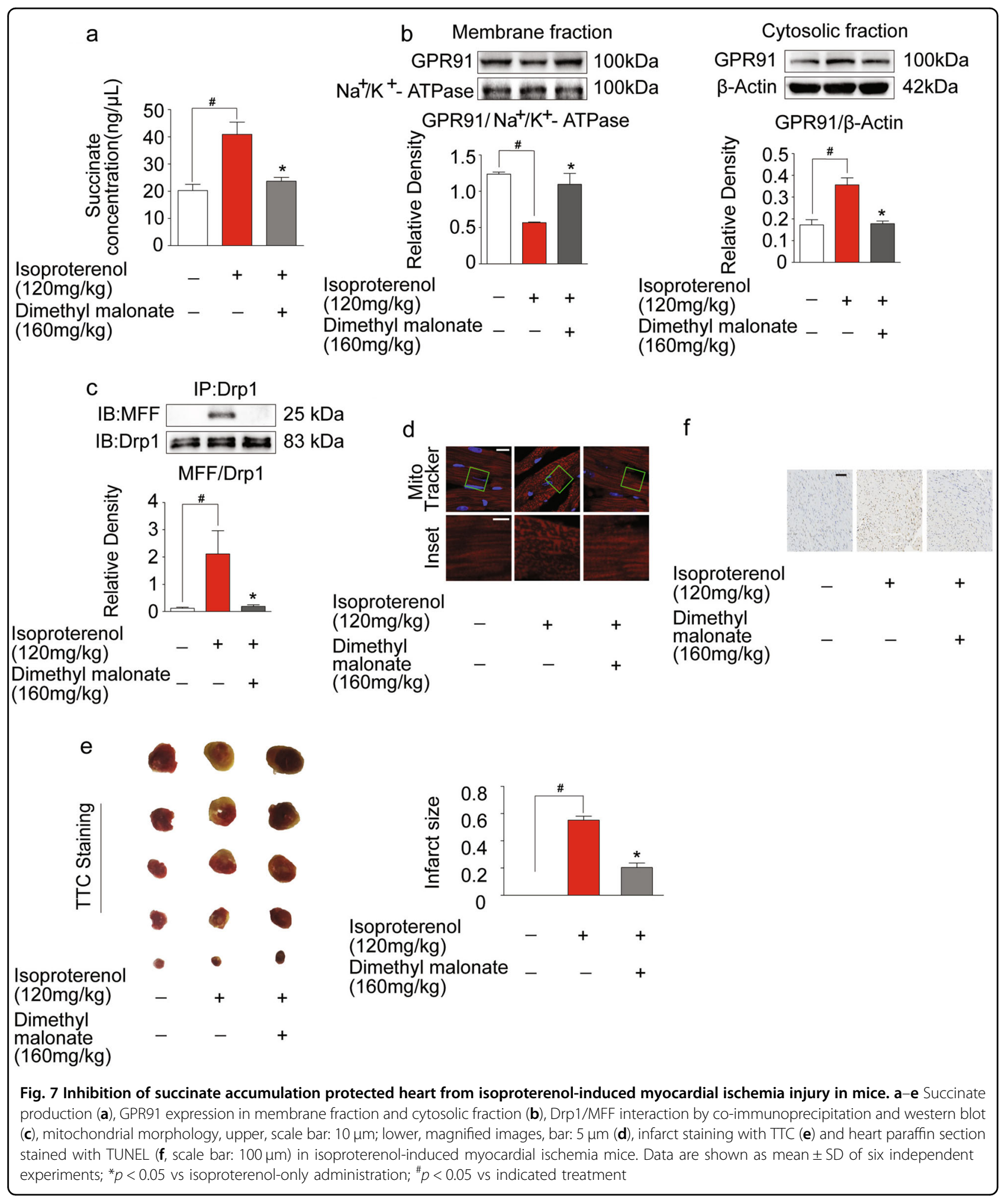

morphology is dynamically controlled by Drp1 and the sub-mitochondrial localization of Drp1 is essential for Drp1 to mediate mitochondrial fission. Two sites are phosphorylated on Drp1 ${ }^{1}$. Phosphorylation of Drp1 at Ser
616 residue in humans, corresponding to Ser 585 in rats, is able to activate Drp1 $1^{21,22}$, while Ser 637 phosphorylation can inhibit Drp1 activation ${ }^{32}$. In the present study, upon succinate stimulation, PKC $\delta$ combined with Drp1 in 
the cytoplasm, leading to Drp1 phosphorylation and then translocation of PKC $\delta$ with phosphorylated Drp1 to mitochondria to induce mitochondrial fission. Knockdown of GRP91 reduced PKC $\delta$ translocation to mitochondria and prevented mitochondrial fission, providing evidence that succinate-mediated GRP91 activation induced mitochondrial fission through regulation of PKC $\delta$. This regulation is consistent with the published study which shows that PKC $\delta$ induced aberrant mitochondrial fragmentation in neuronal cells ${ }^{7}$.

Intriguingly, we found that $\mathrm{PKC} \delta$ but not $\mathrm{PKC} \varepsilon$ is activated in response to GPR91 stimulation. PKC $\delta$ and $\mathrm{PKC} \varepsilon$, the principal members of the novel group of PKCs, are opposed by their principal effects. In the heart, PKCE is favorably involved in the preconditioning protection against ischemic insult, while $\mathrm{PKC} \delta$ serves as a mediator for the ischemic damage ${ }^{33}$. Our previous study also showed that in response to succinate-mediated GPR91 activation, PKC $\delta$ was activated and translocated to mitochondria, impairing pyruvate dehydrogenase activity in cardiomyocytes $^{20}$. Herein, we identified another pathological mechanism by which $\mathrm{PKC} \delta$ activation impairs mitochondrial integrity in cardiomyocytes. The defects in mitochondrial dynamics can impair energy metabolism, leading to mitochondrial dysfunction ${ }^{34}$. Therefore, we reasoned that PKC $\delta$ is involved in both mitochondrial morphological and functional regulation in cardiomyocytes. In fact, PKC $\delta$ has multiple protein substrates. By phosphorylating such diverse substrates at different subcellular locations, PKC $\delta$ mediates a variety of cellular responses ${ }^{35}$.

Upon activation, Drp1 recruits to mitochondria, where it binds to MFF to assemble fission sites. Evidence shows that phosphorylation of MFF by AMPK may increase the association of Drp1 with mitochondria to induce mitochondrial fission ${ }^{9}$. This evidence indicates that MFF phosphorylation facilitates Drp1 binding to mitochondria to influence the dynamic change of mitochondrial morphology. In cardiomyocytes, we showed that MFF contains ERK1/2 consensus phosphorylation sites, indicative of the potency to be regulated by ERK1/2. Importantly, MAPK/ERK signaling is considered to be a downstream regulation by GRP91 activation ${ }^{13,14}$. Succinate-mediated GPR91 activation increased ERK1/2 phosphorylation and promoted ERK1/2 translocation to mitochondria, where it phosphorylated MFF to facilitate Drp1 localization on mitochondria, contributing to mitochondrial fission. Even though it is documented that ERK can directly phosphorylate Drp1 to promote

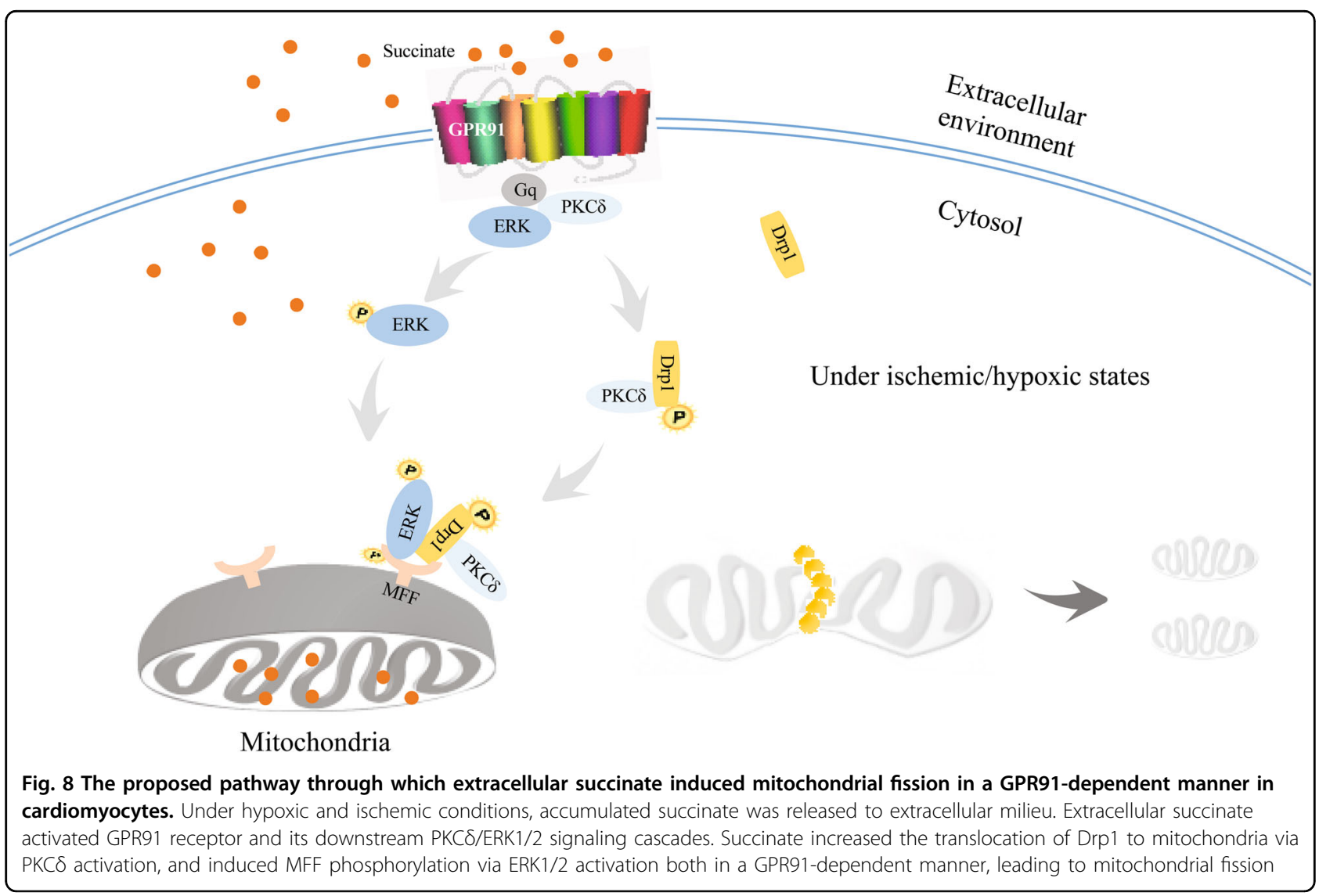


mitochondrial fission ${ }^{8,36,37}$, our work demonstrated another pathway to regulate Drp1 activation by phosphorylation of MFF. Together with the regulation of PKC\&, our study elucidated that PKC/ERK signaling cascades linking GPR91 activation mediated mitochondrial fission upon extracellular succinate stimulation.

Aberrant mitochondrial fragmentation is the early stages of apoptosis ${ }^{38}$. The structural and functional integrity of mitochondria is essential for mitochondrial HK-II binding which is associated with permeability barrier of inner mitochondrial membrane ${ }^{39}$. Succinateinduced mitochondrial fission resulted in the HK-II dissociation from mitochondria, leading to the collapse of mitochondrial membrane potential. Bax and Drp1 colocalize together at scission sites on mitochondria, enabling cytochrome $C$ release from mitochondria ${ }^{40,41}$. We observed that succinate insult increased Bax expression in mitochondrial fraction and promoted cytochrome $C$ release from mitochondria, and subsequently induced cell apoptosis in cardiomyocytes. These alterations were reversed by mitochondrial fission inhibitor mdivi-1, demonstrating that mitochondrial fission contributed to succinate-mediated mitochondrial dysfunction and cell death.

The hypoxic succinate accumulation within cells is a universal metabolic signal in heart ${ }^{42}$, mediating oxidative stress $^{43}$. A recent study shows that accumulated intracellular succinate acts as a potential electron store that fuels to drive ROS formation from mitochondrial complex I by reverse electron transport during reperfusion ${ }^{24}$. Differently, the present work suggested that under hypoxic conditions, excessive extracellular succinate could induce ROS production in response to GPR91-induced mitochondrial fission, which may contribute to exaggerating ROS production to induce oxidative damage during subsequent reperfusion.

To translate our findings in vivo, we observed the protective effects of SDH inhibitor malonate in isoproterenolinduced cardiac ischemia model. We observed that succinate substantially accumulated in the heart and induced GPR91 activation. As expected, SDH inhibitor malonate reduced succinate accumulation in the heart and decreased GPR91 activation against ischemic insult, a regulation likely due to the limited succinate release. Consequently, malonate blocked MFF and Drp1 colocalization in mitochondria and prevented mitochondrial fission. The attenuated heart injury by malonate treatment provided in vivo evidence to support the conclusion that inhibiting succinate-mediated GPR91 signaling may protect the heart from ischemic injury, at least in part.

Taken together, we showed that hypoxic succinate accumulation and release could activate membrane GPR91 and induce mitochondrial fission through PKCס/
ERK1/2 signaling pathways (Fig. 8). These results suggested that inhibition of succinate-mediated GPR91 activation might be a potential therapeutic strategy for protecting cardiomyocytes from ischemic injury.

\section{Acknowledgements}

We thank Xiao-Nan Ma, YingJian Hou and Minhui Sun affiliated to the Cellular and Molecular Biology Center of China Pharmaceutical University for their technical help. We also thank Dr. Ishfaq Muhammad from Northeast Agricultural University for editing the manuscript. This study was supported in part by the National Natural Science Foundation of China (No. 91639115, 81421005, 81573642), Youth Natural Science Foundation of Jiangsu province (No. BK20170750) and Innovation of Graduate Student Training Project in Jiangsu Province (KYLX16_1184)

\section{Conflict of interest}

The authors declare that they have no conflict of interest.

\section{Publisher's note}

Springer Nature remains neutral with regard to jurisdictional claims in published maps and institutional affiliations.

Received: 21 November 2017 Revised: 18 April 2018 Accepted: 10 May 2018

Published online: 04 June 2018

\section{References}

1. Nan, J. et al. Molecular regulation of mitochondrial dynamics in cardiac disease. Biochim. Biophys. Acta 1864, 1260-1273 (2017).

2. Dorn, G. W. Mitochondrial dynamism and heart disease: changing shape and shaping change. EMBO Mol. Med 7, 865-877 (2015).

3. Lesnefsky, E. J., Moghaddas, S., Tandler, B., Kerner, J. \& Hoppel, C. L. Mitochondrial dysfunction in cardiac disease: ischemia--reperfusion, aging, and heart failure. J. Mol. Cell Cardiol. 33, 1065-1089 (2001).

4. Ong, S. B. et al. Inhibiting mitochondrial fission protects the heart against ischemia/reperfusion injury. Circulation 121, 2012-2022 (2010).

5. Yang, Y. L. et al. Ginsenoside Rg5 increases cardiomyocyte resistance to ischemic injury through regulation of mitochondrial hexokinase-ll and dynamin-related protein 1. Cell Death Dis. 8, e2625 (2017).

6. Otera, $\mathrm{H}$. et al. Mff is an essential factor for mitochondrial recruitment of Drp1 during mitochondrial fission in mammalian cells. J. Cell Biol. 191, 1141-1158 (2010).

7. Qi, X., Disatnik, M. H., Shen, N., Sobel, R. A. \& Mochly-Rosen, D. Aberrant mitochondrial fission in neurons induced by protein kinase $C\{d e l t a\}$ under oxidative stress conditions in vivo. Mol. Biol. Cell 22, 256-265 (2011).

8. Kashatus, J. A. et al. Erk2 phosphorylation of Drp1 promotes mitochondrial fission and MAPK-driven tumor growth. Mol. Cell 57, 537-551 (2015).

9. Toyama, E. Q. et al. Metabolism. AMP-activated protein kinase mediates mitochondrial fission in response to energy stress. Science 351, 275-281 (2016).

10. He, W. et al. Citric acid cycle intermediates as ligands for orphan G-proteincoupled receptors. Nature 429, 188-193 (2004).

11. Correa, P. R. et al. Succinate is a paracrine signal for liver damage. J. Hepatol. 47, 262-269 (2007).

12. Sadagopan, N. et al. Circulating succinate is elevated in rodent models of hypertension and metabolic disease. Am. J. Hypertens. 20, 1209-1215 (2007).

13. Lukyanova, L. D. \& Kirova, Y. I. Mitochondria-controlled signaling mechanisms of brain protection in hypoxia. Front Neurosci. 9, 320 (2015).

14. Robben, J. H. et al. Localization of the succinate receptor in the distal nephron and its signaling in polarized MDCK cells. Kidney Int. 76, 1258-1267 (2009).

15. McCreath, K. J. et al. Targeted disruption of the SUCNR1 metabolic receptor leads to dichotomous effects on obesity. Diabetes 64, 1154-1167 (2015).

16. Aguiar, C. J. et al. Succinate modulates $\mathrm{Ca}(2+)$ transient and cardiomyocyte viability through PKA-dependent pathway. Cell Calcium 47, 37-46 (2010). 
17. Aguiar, C. J. et al. Succinate causes pathological cardiomyocyte hypertrophy through GPR91 activation. Cell Commun. Signal. 12, 78 (2014).

18. Simpson, P. \& Savion, S. Differentiation of rat myocytes in single cell cultures with and without proliferating nonmyocardial cells. Cross-striations, ultrastructure, and chronotropic response to isoproterenol. Circ. Res. 50, 101-116 (1982).

19. Sundström, L., Greasley, P. J., Engberg, S., Wallander, M. \& Ryberg, E. Succinate receptor GPR91, a Ga(i) coupled receptor that increases intracellular calcium concentrations through PLCB. FEBS Lett. 587, 2399-2404 (2013).

20. Li, J. et al. Succinate accumulation impairs cardiac pyruvate dehydrogenase activity through GRP91-dependent and independent signaling pathways: therapeutic effects of ginsenoside Rb1. Biochim. Biophys. Acta 1863 2835-2847 (2017).

21. Jahani-Asl, A. \& Slack, R. S. The phosphorylation state of Drp1 determines cell fate. EMBO Rep. 8, 912-913 (2007).

22. Taguchi, N., Ishihara, N., Jofuku, A., Oka, T. \& Mihara, K. Mitotic phosphorylation of dynamin-related GTPase Drp1 participates in mitochondrial fission. J. Biol. Chem. 282, 11521-11529 (2007).

23. Carlson, S. M. et al. Large-scale discovery of ERK2 substrates identifies ERKmediated transcriptional regulation by ETV3. Sci. Signal. 4, rs11 (2011).

24. Chouchani, E. T. et al. Ischaemic accumulation of succinate controls reperfusion injury through mitochondrial ROS. Nature 515, 431-435 (2014).

25. Marín-García, J., Akhmedov, A. T. \& Moe, G. W. Mitochondria in heart failure: the emerging role of mitochondrial dynamics. Heart Fail. Rev. 18, 439-456 (2013).

26. Tannahill, G. M. et al. Succinate is an inflammatory signal that induces $\mathrm{L}-1 \beta$ through HIF-1a. Nature 496, 238-242 (2013).

27. Tretter, L., Patocs, A. \& Chinopoulos, C. Succinate, an intermediate in metabolism, signal transduction, ROS, hypoxia, and tumorigenesis. Biochim. Biophys. Acta 1857, 1086-1101 (2016).

28. Oswald, S., Grube, M., Siegmund, W. \& Kroemer, H. K. Transporter-mediated uptake into cellular compartments. Xenobiotica 37, 1171-1195 (2007).

29. Hems, D. A. \& Brosnan, J. T. Effects of ischaemia on content of metabolites in rat liver and kidney in vivo. Biochem. J. 120, 105-111 (1970).

30. Krebs, H. A. Rate control of the tricarboxylic acid cycle. Adv. Enzyme Regul. 8, 335-353 (1970).
31. Ariza, A. C., Deen, P. M. \& Robben, J. H. The succinate receptor as a novel therapeutic target for oxidative and metabolic stress-related conditions. Front. Endocrinol. (Lausanne) 3, 22 (2012).

32. Li, J. et al. Pharmacological activation of AMPK prevents Drp1-mediated mitochondrial fission and alleviates endoplasmic reticulum stress-associated endothelial dysfunction. J. Mol. Cell Cardiol. 86, 62-74 (2015).

33. Duquesnes, N., Lezoualc'h, F. \& Crozatier, B. PKC-delta and PKC-epsilon: foes of the same family or strangers? J. Mol. Cell Cardiol. 51, 665-673 (2011).

34. Parra, $\vee$. et al. The complex interplay between mitochondrial dynamics and cardiac metabolism. J. Bioenerg. Biomembr. 43, 47-51 (2011).

35. Qvit, N. \& Mochly-Rosen, D. The many hats of protein kinase C 8 : one enzyme with many functions. Biochem. Soc. Trans. 42, 1529-1533 (2014).

36. Kim, B., Park, J., Chang, K. T. \& Lee, D. S. Peroxiredoxin 5 prevents amyloid-beta oligomer-induced neuronal cell death by inhibiting ERK-Drp1mediated mitochondrial fragmentation. Free Radic. Biol. Med 90, 184-194 (2016).

37. Cai, J. et al. ERK/Drp1-dependent mitochondrial fission is involved in the MSCinduced drug resistance of T-cell acute lymphoblastic leukemia cells. Cell Death Dis. 7, e2459 (2016).

38. Yu, T., Sheu, S. S., Robotham, J. L. \& Yoon, Y. Mitochondrial fission mediates high glucose-induced cell death through elevated production of reactive oxygen species. Cardiovasc. Res. 79, 341-351 (2008).

39. Gall, J. M. et al. Hexokinase regulates Bax-mediated mitochondrial membrane injury following ischemic stress. Kidney Int. 79, 1207-1216 (2011).

40. Karbowski, M. et al. Spatial and temporal association of Bax with mitochondrial fission sites, Drp1, and Mfn2 during apoptosis. J. Cell Biol. 159, 931-938 (2002)

41. Montessuit, S. et al. Membrane remodeling induced by the dynamin-related protein Drp1 stimulates Bax oligomerization. Cell 142, 889-901 (2010).

42. Chouchani, E. T. et al. A unifying mechanism for mitochondrial superoxide production during ischemia-reperfusion injury. Cell Metab. 23, 254-263 (2016).

43. Goldberg, N. D., Passonneau, J. V. \& Lowry, O. H. Effects of changes in brain metabolism on the levels of citric acid cycle intermediates. J. Biol. Chem. 241, 3997-4003 (1966). 